\title{
Impacto generado por el COVID-19 en profesionales independientes de Uruguay: caso de estudio
}

\author{
Fecha de recepción: 2021-09-20 • Fecha de aceptación: 2021-11-29 • Fecha de publicación: 2022-01-10
}

\section{Resumen}

El objetivo de este artículo es demostrar el impacto que causó el COVID-19, en diversos profesionales independientes que desempeñan su actividad en el departamento de Maldonado (Uruguay), sí pudieron trabajar como antes o no fue posible, y las maneras que los mismos se ingeniaron para poder superar la pandemia que incide a nivel mundial. De acuerdo a lo anterior, se establece estudiar noticias locales y globales sobre las diversas medidas económicas, financieras, tributarias y laborales abordadas en Uruguay, bibliografía especializada sobre el trabajo independiente y marca profesional; para intentar comprender las diversas facetas que generó la misma situación, las reacciones de estos y formas de sobrepasar la crisis causada por el coronavirus. Como parte de las conclusiones se visualizan diversos comportamientos frente a la misma emergencia, que viene dada por las medidas, por el cierre de los diversos organismos públicos, suspensión de actividades y formas de desempeñar el trabajo.

Palabras clave: ejercicio independiente, branding, marca profesional, crisis sanitaria, Covid-19, coronavirus

\begin{abstract}
The purpose of this article is to demonstrate the impact caused by COVID-19 on various independent professionals working in the department of Maldonado (Uruguay), whether they were able to work as
\end{abstract}


before or not, and the ways in which they managed to overcome the pandemic that affects the world. According to the above, it is established to study local and global news on the various economic, financial, tax and labor measures addressed in Uruguay, specialized bibliography on self-employment and professional branding; to try to understand the various facets that generated the same situation, the reactions of these and ways to overcome the crisis caused by the coronavirus. As part of the conclusions, different behaviors are visualized in the face of the same emergency, which is given by the measures, by the closure of various public agencies, suspension of activities and ways of performing the work.

Keywords: independent practice, branding, professional branding, healthcare crisis, Covid-19, coronavirus 


\section{Introducción}

La globalización ha afectado a los negocios de tres maneras diferentes, por un lado las ventajas competitivas se han fortalecido, por otro, la forma de trabajo frente a la competencia ha variado y, por último, se han generada nuevas oportunidades de negocios (Porter, 2009). La complejidad latente en los mercados hace que sea imposible analizar todas las variables que afectan una situación y poder predecir que va a acontecer en el futuro (Mintzberg et al., 1997). Las crisis son estados constantes para todas las empresas, por eso se debe contar con habilidades sólidas para enfrentarlas (Fener \& Cevik, 2015), éstas van a generar cambios en el qué y cuánto podrán comprar y consumir los clientes (Schiffman et al., 2010).

Adentrarse a los mercados, con la incertidumbre latente, cada vez es más complejo, las empresas deben considerar más factores para poder operar, cómo ser, actuar con transparencia, alcanzar los compromisos adquiridos y conseguir confianza (Covey et al., 2011).

La intención de este artículo es mostrar los problemas y oportunidades de negocios que ha causado el COVID-19, en profesionales independientes de varias profesiones, en el departamento de Maldonado (Uruguay), como también, conocer si los mismos lograron sobreponerse a estos problemas, y las maneras que adoptaron para hacerlo. Lo que se expuso con anterioridad conlleva a la pregunta de investigación: ¿cómo la emergencia sanitaria afectó a los diversos profesionales independientes y qué hicieron para superar esta situación?

En este sentido, el objetivo general en el que se enmarcó este estudio fue el de conocer qué cambios tuvieron los profesionales independientes por causa de la emergencia sanitaria. Este artículo inicia con un marco teórico sobre el ejercicio independiente y elbranding profesional, continúa con las medidas adoptadas por el Gobierno Uruguayo y el efecto en los profesionales. La siguiente sección de la investigación es sobre la metodología que se va usar, continúa con los resultados obtenidos por medio de testimonios de varios profesionales y finalmente se detallan las reflexiones finales y conclusiones del caso de estudio.

\subsection{Marco teórico}

\subsubsection{Medidas adoptadas por el Gobierno}

Posteriormente de confirmarse los primeros 4 casos de COVID-19 en Uruguay, el 13 de marzo de 2020, el presidente Dr. Luis Lacalle Pou anunció la emergencia sanitaria (Presidencia Uruguay, 2020a), en ese momento también se anunció el cierre parcial de fronteras y se suspendieron los espectáculos públicos (Da Silva et al., 2020). A los días se anunció el cierre de museos y la Biblioteca Nacional, como también la suspensión de las clases presenciales por dos semanas, se generó a su vez un teléfono de contacto gratuitos para consultas de la población con médicos, y se le solicitó a la población realizar la menor circulación posible (Presidencia Uruguay, 2020b), las salas de juego de azar y competencias hípicas también se suspendieron (Ministerio de Economía y Finanzas, 2020). Hacia el 16 de marzo se restringieron los vuelos comerciales y se clausuran los puertos deportivos (Presidencia de la República, 2020a). 
Una de las primeras medidas fue brindar subsidio por enfermedad, para quienes debían estar en cuarentena por sospecha de haber contraído COVID-19 (MTSS, 2020a). Hacia el 18 de marzo de 2020 se anunció la creación del subsidio por desempleo parcial para los trabajadores dependientes, que se le reducen las jornadas mensuales o días trabajo; este va a equivaler al $25 \%$ del promedio de las remuneraciones nominales de los últimos meses (MTSS, 2020b), primero tuvo un plazo y al mes siguiente este se amplió. La resolución N 55/020, del 20 de marzo de 2020, autorizó adelantar la licencia generada en 2020 para los trabajadores dependientes.

También, a fines de marzo a los mayores de 65 años que trabajan en relación de dependencia, se les otorgó un subsidio de enfermedad (MTSS, 2020d). La Ley 19.872 de 3 de abril del 2020, anunció que se financia el $60 \%$ de los aportes personales y subsidia el $40 \%$ de los aportes patronales de los trabajadores no dependientes durante el mes de abril y mayo 2020.

\subsubsection{Ejercicio independiente y branding profesional}

La definición de branding profesional fue acuñada por Tom Peters en el año 1997, al día de hoy este concepto ha cobrado mucha relevancia (Cobo \& Arquero, 2017). Las profesiones liberales cumplen con 3 características principales: primero la organización profesional, segundo la independencia del profesional y tercero la capacidad de ser autónomo (Seara, 1960). Para poder construir la marca personal no se necesita una inversión muy elevada; sin embargo, el profesional debe auto-conocerse y definir qué desea lograr al utilizar la marca (Viteri, 2014). Para poder delimitarlo, debe considerar la visión que quiere lograr como profesional y la reputación que tiene frente a los demás, por eso se debe generar huella tanto en el ámbito profesional, como laboral, diseñando un plan de accionar para lograrlo (Rodríguez et al., 2016).

La marca personal va a brindar las herramientas para dar conocer y mostrar el trabajo y contribución al entono, pudiendo de esta manera satisfacer las necesidades de los clientes, implementando valores funcionales y atributos de marca (Altamirano \& Silva, 2018), para lograr el posicionamiento deseado se deben adecuar filosofías coporativas, estrategias empresariales, medios y herramientas (Kotler et al., 2000). Por medio de ello, el branding profesional le brindará las herramientas para que el profesional o su equipo se convierta en la primera opción en la mente del cliente, ofreciendo de esta manera un servicio diferente, visible y relevante (Pérez \& Marcos, 2007), en este sentido, el profesional ha de reinventarse, adoptando nuevas habilidades, situando al cliente en el centro de su estrategia (Suárez, 2018), teniendo en consideración premisas fundamentales como la confianza, buena estima y seguridad (Carreras et al., 2013).

Las empresas son más exitosas cuando despliegan tecnología o la vinculan con los mecanismos tradicionales, considerando el posicionamiento de las mismas (Porter, 2009). Las redes sociales son fundamentales para los profesionales (Viteri, 2014), para ser reconocidos e identificados (Cobo \& Arquero, 2017), los mensajes que se reproducen por estos medios tienen doble función; se puede visualizar la efectividad de los mensajes y por ello se genera una respuesta instantánea por parte de los clientes frente al servicio (Schiffman et al., 2010). Adaptarse a estos modelos es fundamental; dado que se pasó de una generación la cual se comunicaba verbalmente, a una que lo hace de forma visual, por medio de textos, imágenes, etcétera. 


\subsection{Situación de los profesionales independientes y la nueva normalidad}

Los primeros días del mes de abril los profesionales independientes pidieron al Gobierno algún subsidio o exoneración de tributos para superar la situación (El País Uruguay, 2020). La situación es tal, que muchos no podían pagar los aportes, incluso se solicita no perder la cobertura médica, a pesar de no aportar al Fondo Nacional de Salud (en adelante FONASA).

La Caja de Jubilaciones Profesionales prorrogó unas semanas los vencimientos de los aportes del mes de marzo y posteriormente de abril (CJPPU). Lo mismo ocurrió con la Caja Notarial (2020), que también extiende la vigencia de certificados de estar al día con la caja. Los servicios no profesionales están comprendidos en el préstamo "Seguro por cese de actividad" anunciado por el Gobierno; sin embargo, los servicios profesionales no lo están, pero pueden encontrar otras formas de financiamiento.

A fines de abril del 2020, para enfrentar la denominada "nueva normalidad", se anuncia la creación de comité honorario de expertos, quienes van a trabajar en medidas para enfrentar la pandemia (EI Observador, 2020a). Hacia el 5 de mayo de 2020 se anuncia que se están realizando instructivos y protocolos para la vuelta a las actividades (Presidencia de la República, 2020b). El 8 de mayo del 2020 se anunció una flexibilización provisoria y excepcional del seguro de desempleo para los trabajadores dependientes, quienes estén afectados por la emergencia sanitaria; pero que no hayan alcanzado los días para beneficiarse del seguro de desempleo normal (Presidencia Uruguay, 2020c).

\section{Metodología}

La metodología de este artículo es de índole documental, de tipo descriptivo, explicativa, de corte no experimental y transaccional, realizando un estudio de bibliografía y noticias, además de un análisis de testimonios de casos.

Para elaborar el mismo se realizó la investigación desde una perspectiva cualitativa, ya que por medio de esta se intenta visualizar los comportamientos, por medio del relato de experiencias reales. A su vez, es explicativa, ya que busca expresar la situación que atravesaron los diversos profesionales frente a la pandemia, y es descriptiva dado que intenta mostrar características de los hechos suscitados y medidas adoptadas, como también del impacto y continuidad o no de las diversas actividades que desempeñan los profesionales.

De esta manera se desea indagar en la información de testimonios de casos, para posteriormente emitir un juicio, fundamentado en ello, siendo más sencillo para el lector poder comprender los datos.

La elección de la muestra es de carácter no probabilístico, de forma intencional, de acuerdo a los siguientes criterios: primero que sean profesionales que han sido afectados por el COVID-19, segundo que engloben diversas áreas de estudios para complementar el análisis y, a la vez, que estén ejerciendo libremente y de forma independiente su profesión en el departamento de Maldonado (Uruguay). 


\section{Resultados}

A continuación, se presentan una serie de testimonios de profesionales que ejercen libremente en el departamento de Maldonado (Uruguay).

\section{1 Área jurídica y notarial}

\subsubsection{Testimonio 1 - Escribano}

"A nosotros como escribanos la emergencia sanitaria nos afectó completamente el ejercicio de la profesión, dado que los clientes no tenían tantos ingresos, muchos dejaron para regularizar más adelante o no compraban como antes. Con el registro está muy complejo trabajar, aún sigue limitada la capacidad para trabajar, dependemos de la información de los inmuebles y personas (...) dependemos del registro para inscribir los contratos que hacemos, que van solemnizados. Hay un mes de retraso, a las 12 de la noche hay que sacar hora y ya no hay cupo. El sistema de trabajo es totalmente diferente. Si bien podemos firmar el negocio, hasta que no se inscribe y entra al registro, puede tener un embargo. Las sucesiones también fueron complicadas de hacer, porque el poder judicial también cerró. La información de registro de testamentos es compleja poder sacarla, porque también se hace por agenda y hay retrasos. El ejercicio de nuestra profesión se vio muy afectado por la forma en que el registro se adaptó a la emergencia sanitaria, y trabajamos con mucha inseguridad, en mi caso prefiero no firmar documentos que trasladen la propiedad", (comunicación personal, 2 de junio de 2020).

\subsubsection{Testimonio 2 - Doctor en Derecho}

"A nivel judicial se decretó feria judicial sanitaria desde el 16 de marzo al 4 de mayo de 2020, por lo que no se recepcionaban artículos, ni escritos; solo la excepción del área de trabajo penal, menores de edad y violencia doméstica. En este sentido, para los demás trámites y expedientes se congelaron los plazos, ya que no podían ingresar, ni visualizar la situación de los mismos. Solo se trabajaba para dar curso a las emergencias que mencioné. En esos meses estuvo parado totalmente, no podíamos trabajar en nada, en nuestra profesión estuvimos sin ingresos durante ese período, salvo que tuvieras una especialización o te dedicaras al derecho penal y de familia", (comunicación personal, 6 de junio de 2020).

En el ámbito jurídico y notarial estuvieron cerrados los organismos o trabajando focalizado en determinados casos. El trabajo de estos profesionales se vio totalmente comprometido, debido a que su labor depende casi exclusivamente de la operativa de estos organismos, para recibir información, enviar expedientes, poder enviar los escritos, etcétera. La feria judicial extraordinaria por la emergencia sanitaria rigió del 16 de marzo del 2020, al 4 de mayo del 2020 donde se levantó la misma, para comenzar el trabajo de forma progresiva (El Observador, 2020b).

Por lo que a pesar de que tuvieron libertad para desarrollar y ejercer su trabajo, no podían hacerlo o culminarlo al estar los organismos cerrados o trabajando limitadamente por la emergencia sanitaria. 


\section{2 Área diseño y paisajismo}

\subsubsection{Testimonio 3 - Arquitecto}

"Yo soy arquitecta, me dedico a la planificación y diseños de exteriores, mi trabajo no se suspendió, ni tengo contacto asiduo con muchos organismos públicos (salvo la Intendencia o con el Banco de Previsión Social). Cuando surgió la emergencia sanitaria no pude seguir trabajando, los clientes tenían otras prioridades, más allá del arreglo de los exteriores, no sabía qué hacer; dado que mi sueldo depende de mi trabajo realizado. Pero empecé a ingresar a las redes sociales y por medio de ellas vi la oportunidad de fomentar la forma de cultivar plantas y huertas, y cómo organizarlas mejor, qué plantar, cuándo hacerlo, me he especializado en el área y puedo tener ingresos en este sentido. Al tener buena repercusión, empecé a realizar cursos online con asesoramiento y seguimiento de la situación, si bien no es lo mismo que mi trabajo, pude tener algún ingreso para superar la situación”, (comunicación personal, 20 de mayo de 2020).

También frente a las carreras de diseño, de acuerdo a este testimonio, hubo cambios al paralizarse la construcción. Entre el 24 de marzo al 5 de abril de 2020, gremios de la construcción, Sindicato Único Nacional de la Construcción y Afines, con el acuerdo del Ministerio de Trabajo y Seguridad Social, acordaron una licencia especial (MTSS, 2020c), los cambios se suscitan en la forma de desempeñar la carrera, como en la baja actividad causada por la suspensión de actividades e incertidumbre, asimismo, con la exhortación de no salir a la población, cambio de prioridades. En este caso se visualiza claramente que el profesional debió reinventarse para poder trabajar.

\section{3 Área económica y contable}

\subsubsection{Testimonio 4 - Contador público}

"Como contadora, mi trabajo cambió totalmente, no se suspendió, podía trabajar, pero, por ejemplo, los organismos públicos durante la emergencia sanitaria no tenían atención presencial. Entonces cambió la forma de trabajar, lograr que el cliente firme los documentos en la casa y los envíe, para luego escanearlos, fue más complejo poder trabajar en este sentido. Las consultas y reuniones fueron todas vía online. Cuando recién comenzó la pandemia, muchas personas comenzaron a ir al seguro de desempleo y el sistema estaba bastante colapsado, muchas solicitudes daban error y no se sabía las razones y a los teléfonos de contacto era muy complejo poder comunicarse. Frente al seguro de paro parcial, préstamos blandos, subsidios y financiamientos cuando se lanzaron (al ser en medio de la pandemia y no tener mucho tiempo), se veía en las ruedas de prensa y demoraban varios días en quedar activos en los sistemas, lo que generaba dudas y no podía darse una respuesta a los clientes. Hubo cambios también en modalidades, vencimientos y formas en que los clientes pagaban sus tributos. En estos momentos también aproveché para realizar cursos asociados a la experiencia de cliente, estudiando nuevas medidas, y a la vez, para dar cierre a los balances y declaraciones juradas", (comunicación personal, 26 de mayo de 2020),

En el ámbito contable y económico la incertidumbre fue muy grande, ocasionando fuertes cambios en el trabajo y formas de desempeñarlo. Muchas empresas cerraron sus puertas por 
la exhortación, para ver cómo evolucionaba la pandemia, o bien, reducían horario o personal, iba al seguro de desempleo o se le despedía. Durante el primer mes los organismos públicos atendían exclusivamente vía remota; sin embargo, por medio de usuarios y claves web. Es de menester, destacar que los subsidios y seguros de desempleo se intensificaron, lo que claramente complicó la funcionalidad de las páginas y atención. Hacia mayo, algunos comenzaron a abrir de forma presencial, con agenda y protocolos de seguridad, otros a medio horario. Por lo que en un momento surgió incertidumbre sobre si la atención era vía online o presencial. Pero durante la pandemia, de acuerdo al testimonio, se podía llevar a cabo la actividad, con cambios en la modalidad e implementación de las tecnologías de comunicación e información.

\section{4 Área social-humano}

\subsubsection{Testimonio 5 - Licenciado en Psicología}

"En mi caso, como psicólogo trabajo dependiente, cuando comenzó la emergencia sanitaria empecé a trabajar desde casa por mi trabajo. Debido a la incertidumbre latente, muchos conocidos me consultaban por servicios y guía. Por lo que me abrí una empresa unipersonal y empecé a trabajar por ese medio, por servicios de acompañamiento y similares de forma remota. En el caso mío, si bien, las consultas las tenía que cobrar a un menor valor de honorarios, tenía una mayor cantidad de clientes solicitando mis servicios y lo podía hacer por vídeo conferencia y por grupos o similares", (comunicación personal, 10 de mayo de 2020).

En cuanto al ámbito social y psicológico, la incertidumbre latente y el miedo conllevaron a un mayor número de consultas. Lo que ocasionó que los profesionales buscaran la manera de prestar servicios y abarcar a un mayor número de pacientes. Si bien este incremento es en cantidad, los honorarios de acuerdo a los testimonios se redujeron, hasta en algunos casos fue gratuito el servicio.

\section{5 Área salud}

\subsubsection{Testimonio 6 - Licenciado en Enfermería}

"En marzo, cuando comenzó la emergencia sanitaria, no fue un impacto tan elevado para nosotros, pero a medida que pasaban los días, el miedo en la población se fue incrementando. Por suerte, en nuestra policlínica no tuvimos ningún caso positivo; sin embargo, si trasladamos a posibles pacientes. La mayoría de las consultas se hacían de forma telefónica, los llamados los dividimos en clave 1, clave 2 y clave 3 . Siendo el clave 1 el más grave, a los cuales íbamos presencial, los demás se trataban de atender vía telefónica. En principio bajaba sólo el médico, los choferes no bajaban (por lo que tuvimos que cambiar la modalidad de trabajo). Por el distanciamiento nos dividimos, antes viajábamos los 3 adelante, ahora solo el chofer. Cada vez que se traslada un paciente con un síntoma respiratorio tenemos que quedarnos en la sede central, mientras que lavan el móvil. Íbamos al trabajo y cuando volvíamos intentábamos dejar la ropa afuera y usar un pulverizador, evitando la propagación". 
"En la salud fuimos pasando por etapas, risas, susto, pánico por la familia, sentir el rechazo de la gente; a pesar de que no estábamos trabajando. Pero, principalmente se siente muy desprotegido, pasamos mucho miedo e inseguridad. En mi caso tuve que dejar a mi hijo unos días (los de mayor contagio) en la casa de los abuelos, porque cuando iba a trabajar lo tenía que llevar y traer, por lo que me sentía muy sola y lo extrañaba mucho, como él a mí. No me vinculaba tanto con mis familiares, dado que les generaba rechazo que estaba trabajando en la primera línea de contagio. También teníamos problemas para dormir, muchos tomamos medicación. $Y$ es un sentimiento compartido entre todos mis compañeros ahora está volviendo todo a la normalidad", (comunicación personal, 6 de junio de 2020).

\subsubsection{Testimonio 7 - Doctora en Enfermería}

"Lo que me cambió a mí por miedo es la rutina, las precauciones que tomo, cómo ser, bañarme acá en la mutualista, dejar la ropa afuera y desinfectarla. En un primer momento, lo que me pasó fue tener un alto nivel de estrés y de angustia, por mí, mis hijas y mi padre, que vive conmigo. En un principio, las consultas de pacientes aumentaban por la incertidumbre, me sentí colapsada con toda la información que había, y encima era todo nuevo. Y no se sabía cómo operar, era mucha incertidumbre y angustia. Ahora, con todo el camino recorrido, estamos más tranquilos y trabajamos con un poco más de seguridad”, (comunicación personal, 6 de junio de 2020),

Frente a la medicina y la primera línea de contacto, se visualiza un trabajo más fuerte, más duro y con todos los perjuicios que puede causar, cómo ser, más ansiedad, miedos, e insomnio. El miedo fue el gran motor de cambio para el desempeño de la actividad, como también para las consultas que se realizaban.

\subsubsection{Testimonio 8 - Licenciado en Imagenología}

"Yo soy radiólogo, mi trabajo lo cobra a destajo (lo que supone cantidad de pacientes atendidos y estudios realizados), cuando se anunció la emergencia sanitaria la demanda bajo y por ende mi trabajo, ya que solo se hacían estudios y cirugías de urgencia, porque el sistema médico está abocado a superar la emergencia sanitaria por el COVID19 y los clientes están muy preocupados, por lo que evitan hacer estudios que no tengan carácter urgente por la pandemia", (comunicación personal, 28 de mayo de 2020).

\subsubsection{Testimonio 9 - Doctor en Odontología}

"En la profesión de odontología, cuando se decretaron las primeras medidas, estuve parado sin atención durante un mes y medio, en mi área se paralizó todo, se acataron las medidas del Gobierno. Hacia la quinta-sexta semana desde los primeros casos comenzaron las primeras consultas por emergencias. Lo que sí me sirvió el tiempo libre fue para poder contactarme con otros colegas de todo el mundo, sobre todo para la conexión, formación profesional y para acceder a nuevos temas, Webinars, nuevas tecnologías para modelos. Pude adentrarme a la odontología digital, pudiendo trabajar digitalmente con modelos producidos y generados en 3D, para poder imprimirlos y no recurrir a un técnico que haga los modelos a mano". 
"En la "nueva normalidad", en cuanto a la consulta normal nos cambió las barreras de seguridad y el tema de instrumental. Las medidas de barrera se intensificaron mucho, más en el sentido de seguridad, como ser, utilización de sobre-túnica, máscara, doble par de guantes, doble tapaboca, protector fácil de acetato, más otra división especial. Me impactó la forma de trabajar, porque todas estas medidas las uso a diario y me modifica posiciones posturales, trabajo desde una distancia más lejana al paciente, por medio de lupas. Los descartables, desde el punto de vista económicos, han subido su precio considerablemente y es difícil trasladar a los pacientes el costo, la mayor parte de esos gastos lo estoy absorbiendo. Al retomar la actividad, noté que los pacientes tomaron una buena postura, sin notar tanto el impacto, fue como un rebote de pacientes que se estaban conteniendo de no ir a consultas. Por los productos odontólogos y materiales mecánicos, estoy trabajando con encomiendas, pero demoran mucho más que antes", (comunicación personal, 4 de junio de 2020).

En cuanto a la salud, las perspectivas variaban considerablemente, tomando en cuenta las especializaciones y formas de desempeñar las actividades. Frente a las actividades asociadas a odontología o radiología, si bien no se paralizaron las actividades, si bajó considerablemente la operativa, por el miedo y la incertidumbre. Se redujeron los servicios asociados a consultas presenciales y cirugías que no revistieran carácter de urgencia.

\subsection{Síntesis de los resultados obtenidos}

A modo de síntesis de los resultados obtenidos en los testimonios de casos de las diversas profesiones, se obtienen los siguientes resultados que se muestran en la Tabla 1.

Tabla 1.

Síntesis de resultados

\begin{tabular}{|c|c|c|c|c|c|c|c|c|c|}
\hline & T1 & T2 & T3 & T4 & T5 & T6 & T7 & T8 & T9 \\
\hline $\begin{array}{l}\text { No pudo desempeñar su trabajo durante la } \\
\text { pandemia }\end{array}$ & $X$ & $X$ & & & & & & & \\
\hline $\begin{array}{l}\text { Pudo desempeñar su trabajo parcialmente } \\
\text { durante la pandemia }\end{array}$ & & & & & & & & $X$ & $X$ \\
\hline $\begin{array}{l}\text { Tuvo que cambiar la forma de trabajar } \\
\text { durante la pandemia }\end{array}$ & & & $x$ & $X$ & $X$ & & & & \\
\hline $\begin{array}{l}\text { Su trabajo se incrementó y requirió un } \\
\text { mayor control }\end{array}$ & & & & & & $x$ & $x$ & & \\
\hline $\begin{array}{l}\text { En la nueva normalidad volvió a trabajar } \\
\text { como antes, pero, implementando medidas } \\
\text { de seguridad }\end{array}$ & & & & & $x$ & $X$ & $X$ & $X$ & $X$ \\
\hline $\begin{array}{l}\text { En la nueva normalidad, volvió a trabajar } \\
\text { como antes, pero, con más lentitud, } \\
\text { mezclando el ámbito presencial y virtual, } \\
\text { con agendas y medidas de seguridad. }\end{array}$ & $X$ & $X$ & $X$ & $X$ & & & & & \\
\hline $\begin{array}{l}\text { En la época de aislamiento social, indago } \\
\text { nuevas prácticas para poder adoptar su } \\
\text { trabajo y utilizar en un futuro }\end{array}$ & & & $X$ & $x$ & $x$ & & & & $x$ \\
\hline
\end{tabular}


En relación con el objetivo general de la investigación, se puede apreciar que los profesionales independientes del departamento de Maldonado (Uruguay), sufrieron grandes cambios en su forma de trabajo, más allá de su área de experticia, forma de trabajo y de desempeñar sus actividades.

Algunos de ellos tuvieron cambios tan grandes y drásticos que mencionaron que, en períodos de esta pandemia, por causar intrínsecas o extrínsecas a su profesión, o bien, por causas de los organismos con que trabajan, no pudieron hacerlo; lo que le causó grandes pérdidas económicas y laborales. Mientras, que algunos de ellos, tuvieron que re-ingeniarse para adaptar su trabajo, modelo de negocios y poder seguir desempeñando sus actividades.

Otros, en la nueva normalidad, han vuelto a su forma de trabajo incrementando las medidas de higiene y seguridad. Mientras que otros pudieron trabajar de forma parcial por causas variadas, horarios, formas, lugares, etcétera. Algunos mencionan, cómo posible inconveniente, el aumento de controles en su trabajo, lo que ocasiona más demoras para la realización del mismo.

La virtualidad, llegó para quedarse para muchos de ellos, por lo que puede utilizarla como herramienta para mejorar su desempeño, combinado con la virtualidad. Lo mismo ocurrió para otros de ellos, que indagaron nuevas formas de trabajo que pueden aplicar de cara al futuro.

En los momentos de crisis hay que preguntarse ¿qué desean los clientes? y eliminar todo aquello que no entra en la respuesta. Es muy común para las empresas centrarse en los aspectos financieros, en disminución de los clientes, empleados o posicionamiento. Sin embargo, las decisiones se deben basar en lo que el cliente desea y qué es lo que le genera más valor, en el momento de crisis y mayor incertidumbre por la pandemia, aquellos profesionales que pudieron seguir trabajado han tenido grandes cambios en su forma de trabajo, como también, en la planificación estratégica de servicios o modalidades a adoptar en el futuro. Por lo que, muchos de ellos han aprovechado el tiempo para poder reinventarse o adaptar su plan de negocios en ese momento y de cara al futuro.

\section{Conclusiones}

En conclusión, los diferentes profesionales abordaron la situación de emergencia sanitaria de forma distinta, ya sea por medio de las imposibilidades que se le planteó, como ser, feria judicial por la cual no se puede operar, cierre presencial de organismos públicos, algunos han logrado sobreponerse a la situación realizando su trabajo través de una página web, conexión remota o aplicaciones asocias, otros; sin embargo, no pidieron desempeñar su actividad bajo ninguna forma. Al tener postergación de estudios médicos, exhortación a la población de quedarse en casa y evitar aglomeraciones, estos profesionales vieron que su trabajo se pausaba por acciones externas a su actividad, sin lograr que pudieran desempeñar sus actividades normalmente. Otros, vieron afectado su trabajo por cambio de prioridades de los clientes.

Para otros casos no hubo otra alternativa más que la búsqueda de medidas u oportunidades de negocio paralelas o asociadas a su trabajo, para poder obtener otro tipo de ingresos, y poder superar la situación. Estas medidas en el momento fueron necesarias para poder abordar la 
situación, pese a esto, pueden convertirse de cara al futuro en alternativas para poder desempeñar la profesión o complementar los ingresos.

En cuanto a las medidas, las únicas que pudieron adherirse a los profesionales fueron la de postergación de vencimientos de impuestos o aportes a las Cajas Notariales o profesionales. No obstante, podían optar por recibir préstamos por medio de las cajas o bancos, pero no tuvieron tanta variación por la pandemia, como en otros casos. A los seguros de enfermedad, desempleo y desempleo parcial, al ser profesionales independientes, no se pudieron adherir. 


\section{Referencias}

Altamirano, M., \& Silva, J. (2018). Innovación y competitividad de negocios, perspectivas para el desarrollo económico. Ediciones.

Caja de Profesionales Universitarios. (s/f). Nueva posibilidad de prórroga para el pago de aportes. CJPPU. https://www.cjppu.org.uy/novedad.php?id=310

Caja Notarial. (03 de 2020). Prorroga vencimientos. https://www.cajanotarial.org.uy/innovaportal/file/3199/2/ cn-prorroga-de-plazo.jpg

Carreras, E., Alloza, A., \& Carreras, A. (2013). Reputación Corporativa. Editorial empresarial.

Cobo, S., \& Arquero, S. (2017). Análisis de la metodología del Project Management Institute para su aplicación en las Unidades de Información y Documentación. . Actas de las I Jornadas de Estudiantes de Ciencias de la Documentación "Compartiendo Conocimiento", 11.

Covey, S. R., Whitman, B., \& England, B. (2011). Asegurar resultados en tiempos de incertidumbre. Grupo Planeta Spain.

Da Silva, M., Fernández, P., \& Melgar, P. (14 de marzo de 2020). Coronavirus en Uruguay: cierre parcial de fronteras y suspenden espectáculos públicos. El País. https://www.elpais.com.uy/informacion/politica/gobierno-decidio-cierre-relativo-fronteras-confirmacion-coronavirus.html

El Observador. (18 de abril de 2020a). La "nueva normalidad" y su funcionamiento con "pasos cortos". https://www. elobservador.com.uy/nota/la-nueva-normalidad-y-su-funcionamiento-con-pasos-cortos--20204172330

El Observador. (29 de abril de 2020b). A partir del lunes se levanta la feria y vuelven a funcionar los juzgados. https://www.elobservador.com.uy/nota/a-partir-del-lunes-se-levanta-la-feria-y-vuelven-a-funcionar-losjuzgados--2020429133540

El País Uruguay. (16 de abril de 2020). Profesionales independientes solicitan al gobierno la exoneración total de algunos tributos y aportes. https://www.elpais.com.uy/negocios/noticias/profesionales-independientes-solicitan-gobierno-exoneracion-total-tributos-aportes.html

Fener, T., \& Cevik, T. (2015). Leadership in Crisis Management: Separation of Leadership and Executive Concepts. Procedia Economics and Finance, 26, 695 - 701. https://doi.org/10.1016/S2212-5671(15)00817-5

Kotler, P., Camara, D., Grande, I., \& Cruz, I. (2000). Dirección de Marketing. Pearson Educación. 
Ministerio de Economía y Finanzas. (15 de marzo de 2020). Cierre de salas de juegos de azar administradas por la Dirección General de Casinos y suspensión de competencia hípica. https://www.gub.uy/ministerio-economia-finanzas/comunicacion/noticias/cierre-salas-juegos-azar-administradas-direccion-general-casinos-suspension

Ministerio de Trabajo y Seguridad Social. (16 de marzo de 2020a). Subsidio por enfermedad para quienes deban permanecer en cuarentena. MTSS. https://www.gub.uy/ministerio-trabajo-seguridad-social/comunicacion/noticias/subsidio-enfermedad-para-deban-permanecer-cuarentena

Ministerio de Trabajo y Seguridad Social. (18 de marzo de 2020b). Subsidio especial para actividad privada por efectos de COVID-19. MTSS. https://www.gub.uy/ministerio-trabajo-seguridad-social/comunicacion/ noticias/subsidio-especial-para-actividad-privada-efectos-covid-19

Ministerio de Trabajo y Seguridad Social. (23 de marzo de 2020c). Construcción: Licencia especial comienza el 24 de marzo. MTSS. https://www.gub.uy/ministerio-trabajo-seguridad-social/comunicacion/noticias/construccion-licencia-especial-comienza-24-marzo

Ministerio de Trabajo y Seguridad Social. (29 de mayo de 2020d). Se prorrogó el subsidio por enfermedad para trabajadores de 65 años o más. MTSS. https://www.gub.uy/ministerio-trabajo-seguridad-social/comunicacion/noticias/se-prorrogo-subsidio-enfermedad-para-trabajadores-65-anos

Mintzberg, H., Quinn, J., \& Voyer, J. (1997). El proceso estratégico: conceptos, contextos y casos. Edición Breve. Pearson Educación Mexico.

Pérez, A., \& Marcos, T. (2007). ¿Quién teme al Personal Branding? Capital Humano, (210), 94-106. http://andresperezortega.com/documentos/capitalhumano.pdf

Porter, M. (2009). Ser competitivo. Ediciones Deusto.

Presidencia de la República. (16 de marzo de 2020a). Informe de situación sobre coronavirus COVID-19 en Uruguay (16/03/2020). Sistema Nacional de Emergencias. https://www.gub.uy/sistema-nacional-emergencias/comunicacion/comunicados/informe-situacion-sobre-coronavirus-covid-19-uruguay-16032020

Presidencia de la República. (05 de mayo de 2020b). Informe de situación en relación al coronavirus COVID-19 en Uruguay del 05/05/2020. https://www.gub.uy/sistema-nacional-emergencias/comunicacion/comunicados/informe-situacion-relacion-coronavirus-covid-19-uruguay-del-05052020

Presidencia Uruguay. (14 de marzo de 2020a). Gobierno declaró emergencia sanitaria por coronavirus y anunció las primeras medidas. https://www.gub.uy/presidencia/comunicacion/noticias/gobierno-declaro-emergencia-sanitaria-coronavirus-anuncio-primeras-medidas 
Presidencia Uruguay. (15 de marzo de 2020b). Gobierno definió suspensión de clases en centros educativos públicos y privados de todo el país por próximos 14 días. https://www.gub.uy/presidencia/comunicacion/ fotos/gobierno-definio-suspension-clases-centros-educativos-publicos-privados-todo

Presidencia Uruguay. (09 de mayo de 2020c). Flexibilización provisoria y excepcional de acceso al seguro de desempleo beneficiará a trabajadores afectados por emergencia sanitaria. https://www.gub.uy/presidencia/comunicacion/noticias/flexibilizacion-provisoria-excepcional-acceso-seguro-desempleo-beneficiara

Rodríguez, C., Antonio, J., \& Navarro, Y. (2016). Nuevos retos en orientación aboral: de itinerarios personales de inserción a la construcción de marcas profesionales. REOP - Revista Española de Orientación y Psicopedagogía, 27(2), 126-133. https://doi.org/10.5944/reop.vol.27.num.2.2016.17148

Schiffman, L., Lazar, L., \& Wisenblit, J. (2010). Comportamiento del consumidor. Editorial Pearson.

Seara, L. (1960). La independencia de las profesiones liberales. Revista de estudios políticos, (113), 147-158.

Suárez, M. (2018). Buenas prácticas comunicacionales del contador público. Estudio de casos en micro y pequeñas empresas. Revista iberoamericana de contabilidad de gestión, 16(32), 42-53.

Viteri, K. (2014). Establecimiento de un modelo de branding personal en redes sociales para un ingeniero geólogo, que desarrolla sus actividades profesionales a nivel nacional en Ecuador. [Tesis de grado, Pontificia Universidad Católica del Ecuador]. Repositorio Puce. http://repositorio.puce.edu.ec/handle/22000/11699 
Copyright (c) 2022 María Macarena Suárez Blanco

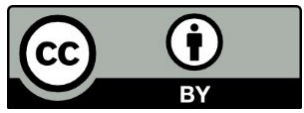

Este texto está protegido bajo una licencia internacional Creative Commons 4.0.

Usted es libre para Compartir-copiar y redistribuir el material en cualquier medio o formato - y Adaptar el documento - remezclar, transformar y crear a partir del material-para cualquier propósito, incluso para fines comerciales, siempre que cumpla las condiciones de Atribución. Usted debe dar crédito a la obra original de manera adecuada, proporcionar un enlace a la licencia, e indicar si se han realizado cambios. Puede hacerlo en cualquier forma razonable, pero no de forma tal que sugiera que tiene el apoyo del licenciante o lo recibe por el uso que hace de la obra.

\section{$\underline{\text { Resumen de licencia - Texto completo de la licencia }}$}

